\title{
Optimal control of quantum systems by chirped pulses
}

\author{
Amstrup, Bjarne; Doll, J. D.; Sauerbrey, R. A.; Szabó, G.; Lorincz, A.
}

Published in:

Physical Review A

Link to article, DOI:

10.1103/PhysRevA.48.3830

Publication date:

1993

Document Version

Publisher's PDF, also known as Version of record

Link back to DTU Orbit

Citation (APA):

Amstrup, B., Doll, J. D., Sauerbrey, R. A., Szabó, G., \& Lorincz, A. (1993). Optimal control of quantum systems by chirped pulses. Physical Review A, 48(5), 3830-3836. https://doi.org/10.1103/PhysRevA.48.3830

\section{General rights}

Copyright and moral rights for the publications made accessible in the public portal are retained by the authors and/or other copyright owners and it is a condition of accessing publications that users recognise and abide by the legal requirements associated with these rights.

- Users may download and print one copy of any publication from the public portal for the purpose of private study or research.

- You may not further distribute the material or use it for any profit-making activity or commercial gain

- You may freely distribute the URL identifying the publication in the public portal

If you believe that this document breaches copyright please contact us providing details, and we will remove access to the work immediately and investigate your claim. 


\title{
Optimal control of quantum systems by chirped pulses
}

\author{
B. Amstrup* \\ Institute of Isotopes of the Hungarian Academy of Sciences, P.O. Box 77, H-1525 Budapest, Hungary \\ and Chemistry Department B, The Technical University of Denmark, DTH-301, DK-2800 Lyngby, Denmark \\ J. D. Doll \\ Department of Chemistry, Brown University, Providence, Rhode Island 02912 \\ R. A. Sauerbrey \\ Department of Electrical Engineering, Rice University, P.O. Box 1892, Houston, Texas 77251 \\ G. Szabó \\ Department of Optics and Quantum Electronics, József Attila University, Dóm tér 9, H-6720 Szeged, Hungary
}

A. Lőrincz ${ }^{\dagger}$

Institute of Isotopes of the Hungarian Academy of Sciences, P.O. Box 77, H-1525 Budapest, Hungary

(Received 29 April 1993)

\begin{abstract}
Research on optimal control of quantum systems has been severely restricted by the lack of experimentally feasible control pulses. Here, to overcome this obstacle, optimal control is considered with the help of chirped pulses. Simulated annealing is used as the optimizing procedure. The examples treated are pulsed population inversion between electronic levels, and optimization of vibronic excitation in the presence of another electronic level. In the problem of population inversion effective potentials of displaced harmonic oscillators are used. For optimizing vibronic excitation the CsI model potential is used.
\end{abstract}

PACS number(s): $33.80 .-\mathrm{b}, 42.50 . \mathrm{Hz}$

\section{INTRODUCTION}

Much effort has been put into developing methods for controlling chemical reactions and/or molecular excitation via the manipulation of laser fields (see, e.g., [1-35]). In many of these computations the problem was the efficiency of the optimal control scheme. It is now established that light shaping is an efficient way of controlling quantum systems and thus, for example, chemical reactions. These theoretical results will presumably give a push to the progress of laser technology, since the optimal pulses indicated in most of these publications are way beyond present-day possibilities.

Here we try to focus on the experimental possibilities. Present-day femtosecond laser technology is viewed as follows.

(1) It is possible to create a short pulse or two related short pulses in the laboratory.

(2) The duration of the pulse or the pulses cannot be shorter than $30 \mathrm{fs}$ if long-term reliability is required.

(3) It is possible to adjust the phase of a "two-pulse sequence" (see, e.g., [36]).

(4) Pulse shaping is troublesome if (i) shaping involves the amplitude and the phase of the field as a function of time and (ii) the required time resolution for shaping is a few femtoseconds or below, as suggested by most results of optimal control computations (see, e.g., $[5,15]$ ). New and flexible solutions have been appearing on this field, however, that use liquid-crystal technology or holographical methods $[37,38]$. 20-fs pulse shaping has been demonstrated already [39]. Further advances of these technologies may be able to create pulse shapes suggested in the literature.

(5) The experimental technology of pulse shaping has opened another direction, the direction of chirped pulses. In other words, the approach of "chirp expansion" is nowadays feasible up to at least third order [40]. This route has attractive properties also since the spectral width of present-day chirped pulses is very broad (it corresponds to a few-femtoseconds pulse length), allowing a broad range of quantum states to contribute in the control process.

Here we deal with the power of a single chirped pulse in optimal control. The results are promising especially if one considers that point (1) states that two pulses are feasible as well as noting the mixing of two pulses is a feasible route for creating more complex electric-field shapes [41].

In the theoretical description of the control problem we use several approximations, e.g., we use BornOppenheimer (BO) surfaces in one case and displaced harmonic oscillators as model potentials in the other. Since any of the approximations may be crude, what can one really say to the experimentalists? Since this range of research may be oriented towards chemical technology, then what can we say to the engineers? If, however, we were to design an optimal control procedure, 
then we should note that the experiment is at the edge of present-day technology, the control unit is subject to aging, the parameters of the procedure, such as temperature, may change, the optical components may move, electrical components may drift, etc. All of these uncertainties should direct our attention towards adaptive control systems. It is important to underline that optimal control through chirped pulses may be made adaptive through on-line control, since the elements one controls here are the positions and the directions of prisms and gratings $[40,42]$. The first approach along this route was suggested by Judson and Rabitz through the use of a genetic algorithm [16] (see also [20]). In the chirped-pulse approach one might try to consider the method that we shall use for optimization, namely the method of simulated annealing.

We have decided to use simulated annealing for three reasons.

(1) "Chirp expansion" does not favor gradient methods since the gradient becomes complicated.

(2) The small number of parameters in chirp expansion makes simulated annealing possible.

(3) In contrast to a gradient method, simulated annealing can guarantee that the search ends in the global maximum $[43,44]$.

There are two problems we shall treat here: that of population inversion and that of generating largeamplitude ground-state vibrational coherence [45]. With regard to population inversion two displaced harmonic oscillators are used as a model system. For comparison, this problem is considered with the help of electric fields of a few Fourier components and also with chirped pulses. The results presented here are promising and show efficient control with the help of chirped pulses. However, it is known that the transition dipole moment is a function of the rotational quantum number [46] and that influences the solution of the optimal control approach [19]. The results from the latter work show that optimal control is feasible for the general case as well at the expense of more intense electric fields.

\section{OPTIMAL CONTROL APPROACH}

A simple model is treated in which there are only two electronic states in the process under control. The equation of motion in our system is the time-dependent
Schrödinger equation $\left(\partial_{t} \equiv \frac{\partial}{\partial t}\right)$

$$
i \hbar \partial_{t} \Psi(\mathbf{x}, t) \equiv i \hbar \dot{\Psi}(\mathbf{x}, t)=\widehat{H} \Psi(\mathbf{x}, t)
$$

In the semiclassical and adiabatic approximation,

$$
\widehat{H}=\left(\begin{array}{cc}
\widehat{H}_{g} & -\boldsymbol{\mu}_{u g} \cdot \mathcal{E}(t) \\
-\boldsymbol{\mu}_{g u} \cdot \mathcal{E}(t) & \widehat{H}_{u}
\end{array}\right)
$$

where $\mu_{g u}$ denotes the matrix element of the dipole operator $\mu$ between the electronic wave functions, and the nuclear wave functions are described by the vector

$$
\Psi=\left(\begin{array}{c}
\psi_{g} \\
\psi_{u}
\end{array}\right)
$$

The subscript $g$ refers to the "ground"-state potential energy surface and $u$, correspondingly, to the "upper" state potential-energy surface. $\widehat{H}_{i}=\widehat{T}_{i}+V_{i}(\mathbf{x})$, where $i=g, u$; $\widehat{T}_{i}=\hat{p}^{2} / 2 m$ is the kinetic-energy operator and $V_{i}$ is the corresponding potential energy. $\mathcal{E}(t)$ represents the amplitude of the classical electric field. The electric field is a physical quantity and is represented by a real function. We consider two types of electric fields, both of which offer a broad spectrum, have a low number of parameters to be adjusted (thus enabling simulated annealing), and look attractive from an experimental point of view. The first of these is given by a Fourier-series expansion in a given time interval with a base frequency $\omega_{0}$ (here we considered ten terms in the expansion):

$$
\begin{aligned}
\mathcal{E}(t)=\sum_{k=0}^{9}\{ & A_{k} \sin \left[\left(\omega_{0}+k \delta \omega\right) t\right] \\
& \left.+B_{k} \cos \left[\left(\omega_{0}+k \delta \omega\right) t\right]\right\} \sin ^{2}(\pi t / \tau) .
\end{aligned}
$$

The terms in this sum have the same sine squared envelope function.

The second type of electric field is the chirp expansion, which could result from propagation through matter, such as fiber optics, and may be manipulated $[40,47,48]$. We have chosen the following form to express the chirp [42]:

$$
\begin{aligned}
\mathcal{E}(t)= & \operatorname{Re}\left\{A \int \mathcal{E}_{0}(\omega) \exp [-i \beta(\omega) z] e^{i \omega t} d \omega\right\} \\
= & \operatorname{Re}\left\{A \int \mathcal { E } _ { 0 } ( \omega ) \operatorname { e x p } \left[-i \beta\left(\omega_{0}\right) z-i \beta^{\prime}\left(\omega_{0}\right) z\left(\omega-\omega_{0}\right)\right.\right. \\
& \left.\left.-\left(\frac{\tau}{4}+\frac{i}{2} \beta^{\prime \prime}\left(\omega_{0}\right) z\right)\left(\omega-\omega_{0}\right)^{2}-\frac{i}{6} \beta^{\prime \prime \prime}\left(\omega_{0}\right) z\left(\omega-\omega_{0}\right)^{3}\right] e^{i \omega t} d \omega\right\}
\end{aligned}
$$


Here $\operatorname{Re}$ denotes the real part and $\mathcal{E}_{0}(\omega)$ is the frequency spectrum of a Gaussian pulse before entering the dispersive medium,

$$
\mathcal{E}_{0}(t)=\operatorname{Re}\left\{A \exp \left(-t^{2} / \tau+i \omega_{0} t\right)\right\}
$$

that is,

$$
\mathcal{E}_{0}(\omega)=A \sqrt{\frac{\tau}{2}} e^{-\tau\left(\omega-\omega_{0}\right)^{2} / 4}
$$

$z$ is the thickness of the dispersive medium and $\beta(\omega)$ is the frequency-dependent propagation factor if the chirp is to be created by direct propagation. On the righthand side of (2.5) we have applied a Taylor expression of $\beta(\omega)$ around the central frequency $\omega_{0}$ prior to entering the dispersive medium, up to the third power of $\left(\omega-\omega_{0}\right) . \beta\left(\omega_{0}\right)$ is merely a phase factor, $\beta^{\prime}\left(\omega_{0}\right)$ gives a group delay, $\beta^{\prime \prime}\left(\omega_{0}\right)$ is part of the chirp as well as pulse (de)compression. The third derivative, which is not usually considered, but could be adjusted if required [40], may give rise to some beating in the pulse shape. For our purposes, the expansion factors $\beta^{(n)}$ where $n$ denotes the $n$th derivative multiplied with thickness $z$, may be considered as the set of parameters to be optimized.

The objective of the optimal control, $\bar{I}_{\text {inv }}$, of population inversion is:

$$
\bar{I}_{\mathrm{inv}}=\left\langle\psi_{u}\left(t_{f}\right) \mid \psi_{u}\left(t_{f}\right)\right\rangle-\lambda \int_{t_{0}}^{t_{f}} \mathcal{E}(t)^{2} d t
$$

where the first term is the population on the upper surface at the final time $t_{f}$. The second term is a penalty term to limit the energy of the electric field and $\lambda$ is a penalty factor. The electric field of this treatment should not have a discontinuity due to the form of the envelope function and there is no need to include a "weight function" as in [19].

The objective of the optimal control of generating ground-state vibrations is

$\bar{I}_{\text {CsI }}=\sum_{j=3}^{8}\left|\left\langle\varphi_{j} \mid \psi_{g}\left(t_{f}\right)\right\rangle\right|^{2}-\lambda \int_{0}^{250 \mathrm{fs}} \mathcal{E}^{2}(t) d t$

where $\varphi_{j}$ denotes the $j$ th vibrational eigenstate of the ground electronic surface of CsI. The eigenstates are calculated by the relaxation method of Kosloff and Tal-Ezer [49].

In the simulated annealing procedure a new electric field is generated at every step with the help of random modification of the parameters. Then a new "annealing" objective is defined as

$$
I_{\text {anneal }}=1-\bar{I}
$$

for the minimum search in the annealing procedure. The new electric field is then tested by determining whether

$$
x>\exp \left(\Delta I_{\text {anneal }} / T\right),
$$

where $x$ is a random number between 0 and $1, \Delta I_{\text {anneal }}$ is the difference between the new and the old annealing objectives, and $T$ is the parameter of the procedure-the "temperature" if the objective corresponds to an energy expression. If one approaches zero with the analog of temperature sufficiently slowly, it guarantees that we end up in the global maximum with probability 1 . The conditions for the suitable rate of temperature change may be determined [50].

The optimization procedure was applied to the following problems: (1) nondisplaced harmonic BO surfaces and an electric field given by (2.4) where the set of $\left(A_{k}, B_{k}\right)$ is to be optimized to invert population (test example); (2) displaced harmonic BO surfaces and an electric field given by $(2.4)$ where the set of $\left(A_{k}, B_{k}\right)$ is to be optimized to invert population; (3) displaced harmonic $\mathrm{BO}$ surfaces and an electric field given by (2.5) where the set of $\left(A, \beta\left(\omega_{0}\right) z, \beta^{\prime}\left(\omega_{0}\right) z, \beta^{\prime \prime}\left(\omega_{0}\right) z, \beta^{\prime \prime \prime}\left(\omega_{0}\right) z\right)$ is to be optimized to invert population; and (4) ground and excited electronic states of CsI and an electric field given by (2.5) where the set of $\left(A, \omega_{0}, \beta\left(\omega_{0}\right) z, \beta^{\prime}\left(\omega_{0}\right) z, \beta^{\prime \prime}\left(\omega_{0}\right) z, \beta^{\prime \prime \prime}\left(\omega_{0}\right) z\right)$ is to be optimized to increase vibrations in the ground electronic state by going through the excited electronic state.

\section{RESULTS}

The time-dependent Schrödinger equation was solved numerically by a split operator method $[51,52]$ that uses the grid method and fast Fourier transformation for computing the Laplacian operator.

All calculations were carried out using atomic units, that is, $1 E_{H}=219474.64 \mathrm{~cm}^{-1}, 1 \hbar / E_{H}=0.0241888 \mathrm{fs}$, $1 E_{H} /\left(e a_{0}\right)=5.14221 \times 10^{11} \mathrm{~V} / \mathrm{m}$, where $E_{H}$ denotes the Hartree energy.

\section{A. Inversion problem}

The parameters were the same as in [19]: a reduced mass equal to $80000 m_{e}$, and the frequencies were identical on both surfaces with the value $5 \times 10^{-4}$ a.u. The displacements are $2.53 \sigma$ for cases with displaced surfaces, where $\sigma=\sqrt{\hbar /(M \omega)}$ is the position uncertainty of the lowest-energy vibrational state. The initial wave function is the lowest vibrational eigenstate of the lower surface. The optimizations were all restricted to pulses with a full width at half maximum (FWHM) of $50 \mathrm{fs}$. Thus, the calculations with sine squared envelope had a total time period of $100 \mathrm{fs}$. Due to the long tails of the Gaussian fields, the calculations with the chirped pulses were initially on a $300-\mathrm{fs}$ time scale. This time interval was lengthened to $450 \mathrm{fs}$ at a later stage as was required by the optimal chirp parameters. The results of the calculations are given in Table I.

The optimized electric fields of the population inversion problem are shown in Fig. 1. In this figure we also give the probability density of the final time wave functions on the ground (small amplitude, dashed lines) and excited (solid lines) surfaces, too.

For nondisplaced potentials perfect population inversion is possible and the exact result for a sine squared envelope function is a resonant field with amplitude $A-$ 
TABLE I. Results of inversion optimizations.

\begin{tabular}{|c|c|c|c|c|c|c|}
\hline$\Delta R_{0} / \sigma$ & $\lambda$ & $\omega_{0}$ & $\delta \omega$ & $\left\langle\psi_{u}\left(t_{f}\right) \mid \psi_{u}\left(t_{f}\right)\right\rangle$ & $\bar{I}$ & $\bar{I}_{\mathrm{ini}}$ \\
\hline \multicolumn{7}{|c|}{ Unchirped } \\
\hline 0 & 50 & 0.0494 & 0.0002 & 0.997 & 0.919 & 0.845 \\
\hline 2.53 & 20 & 0.0506 & 0.0003 & 0.954 & 0.879 & 0.727 \\
\hline 2.53 & 2 & 0.0506 & 0.0003 & 0.973 & 0.962 & 0.648 \\
\hline \multicolumn{7}{|c|}{ Chirped } \\
\hline 2.53 & 2 & 0.0515 & & 0.975 & 0.968 & 0.727 \\
\hline
\end{tabular}
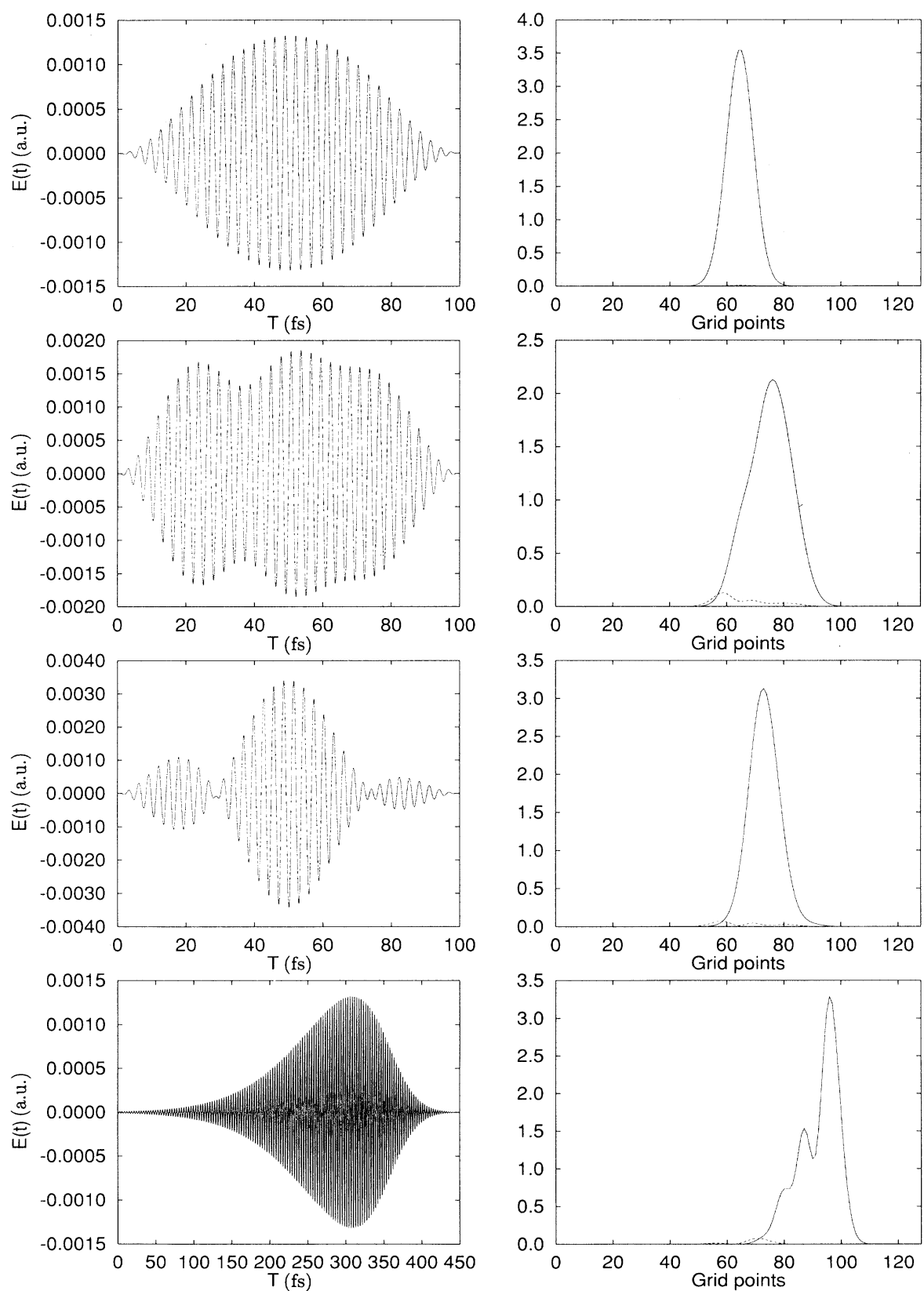

FIG. 1. Optimized electric fields (left) and the wave functions numerically squared on the upper surface and the lower surface (dashed line). The figures at the top are for undisplaced potentials and ten Fourier components. Figures in the second and third rows from the top are for displaced potentials and ten Fourier components. The values of $\lambda$ are -20 and -2 , respectively. The figures at the bottom are with chirped pulse and displaced potentials. 
this amplitude being determined through the so-called area condition

$$
A \int_{0}^{\tau} \sin ^{2}(\pi t / \tau) d t=\frac{1}{2} A \tau=\pi,
$$

that is, the "perfect" population inverting electric field is given as

$$
\frac{2 \pi}{\tau} \sin ^{2}(\pi t / \tau) \cos \left(\omega_{0} t\right), 0 \leq t \leq \tau
$$

where $\omega_{0}$ is the resonance frequency. The target value for this $\pi$ pulse may be determined as 0.910 using a penalty factor $\lambda=50$. The target value of the result of the simulated annealing procedure was somewhat larger than this value and much larger than for our initial "guess" for the electric field that gave 0.845 . The population inversion was 0.997 , which is somewhat smaller than the theoretical maximum of 1.0. The procedure was then stopped as convergence at this point became very slow and gave rise to changes of less than 0.00001 .

The second and the third graphs show the results for the displaced case and the Fourier series approach. The difference between these runs was in our "policy" on the cost of the electric field energy. For the second case $\lambda=20$ and for the third case $\lambda=2$. There is no direct correlation between the penalty factor and the amplitude of the electric field, but the amplitude of the $\lambda=20$ case is definitely smaller. The parameters found by the simulated annealing gave rise to a smoother envelope. According to present-day laser technology it is not a problem to create any of these electric-field amplitudes. We tried to use a strict policy on the electric-field energy to stay within the validity of the two electronic surface approximation. The larger the electric-field amplitude, the worse the approximation. It may be seen that the $\lambda=2$ case resulted in a broader spectrum and sharper features that could better invert the population. The population inversion becomes perfect if the excited-state wave func-
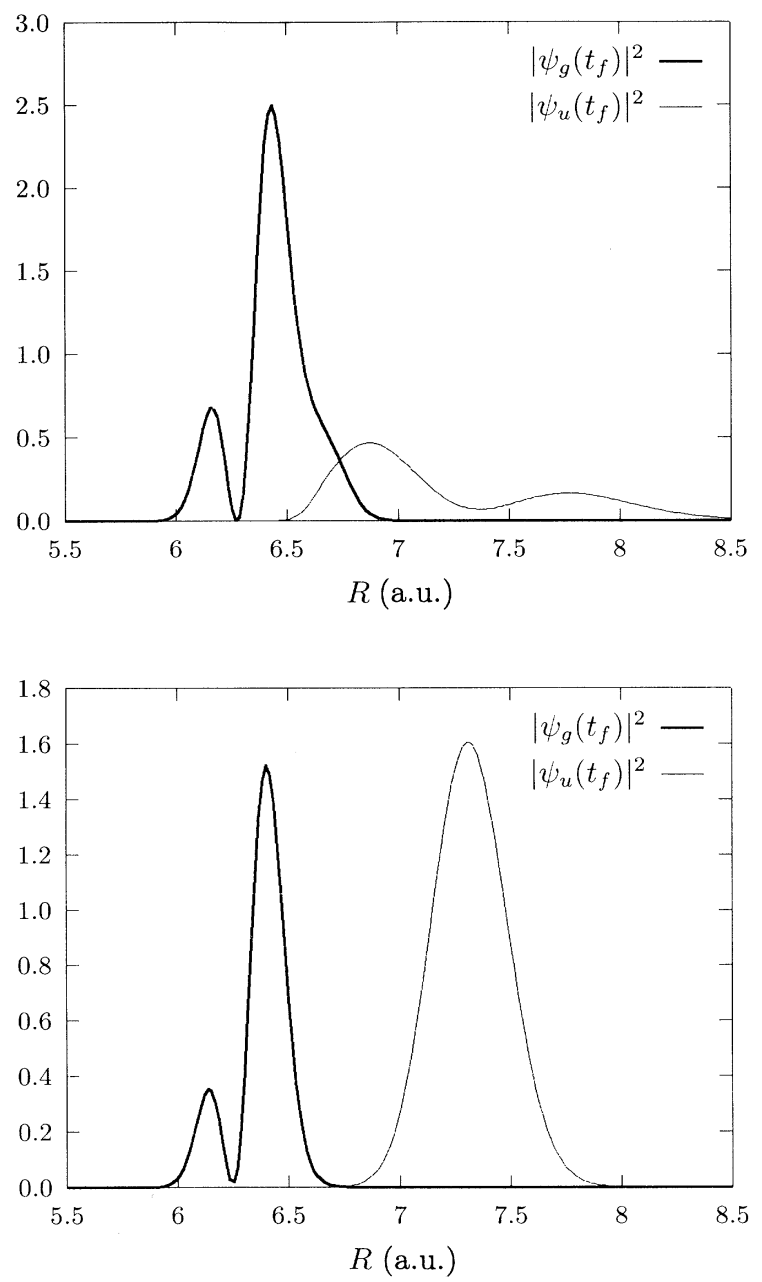

FIG. 2. CsI wave function at final time with optimized chirped pulse (upper graph) and with the same pulse except for chirping parameters set to 0 (lower graph).

TABLE II. Parameters of potentials and grid used in the simulation of CsI dynamics (as given

\begin{tabular}{|c|c|}
\hline Ground potential & Excited potential \\
\hline $\begin{array}{l}V_{g}=a \exp (-\beta r)-1 /\left(r-r_{1}\right) \\
a=131.569 \text { a.u. } \\
\beta=1.37042 \text { a.u. } \\
r_{1}=0.893631 \text { a.u. }\end{array}$ & $\begin{array}{l}V_{u}=\exp \left[-d\left(r-r_{e}\right)\right]-C_{6} /\left(r-r_{2}\right)^{6}+V_{\infty} \\
d=2.57102 \text { a.u. } \\
r_{e}=4.20 \text { a.u. } \\
C_{6}=10.0 \text { a.u. } \\
r_{2}=1.88972 \text { a.u. } \\
V_{\infty}=-0.02939921 \text { a.u. }\end{array}$ \\
\hline \multicolumn{2}{|c|}{ Dipole moment } \\
\hline \multicolumn{2}{|l|}{ Masses } \\
\hline$m_{\mathrm{Cs}}=132.909 \mathrm{amu}$ & $m_{\mathrm{I}}=126.904 \mathrm{amu}$ \\
\hline \multicolumn{2}{|l|}{ Grid } \\
\hline$N=256$ & $\Delta r=0.0165$ a.u. \\
\hline
\end{tabular}
in $[45])$. 
tion cannot move during excitation; in other words, it becomes perfect with a Dirac $\delta$ function at infinite cost. The $\lambda=20$ case resulted in a 0.954 inversion value, while the less strict case gave a 0.973 value. The corresponding target values were 0.880 and 0.963 , respectively.

The last set of graphs corresponds to chirped-pulse population inversion and $\lambda=2$. The change of the shape of the envelope indicates that the third derivative in the chirp expansion plays a role here. The inversion value of 0.981 could be improved by decreasing the penalty on the electric-field energy.

\section{B. CsI calculations}

For vibronic excitation of CsI we present the results of three different computations. The parameters of the CsI
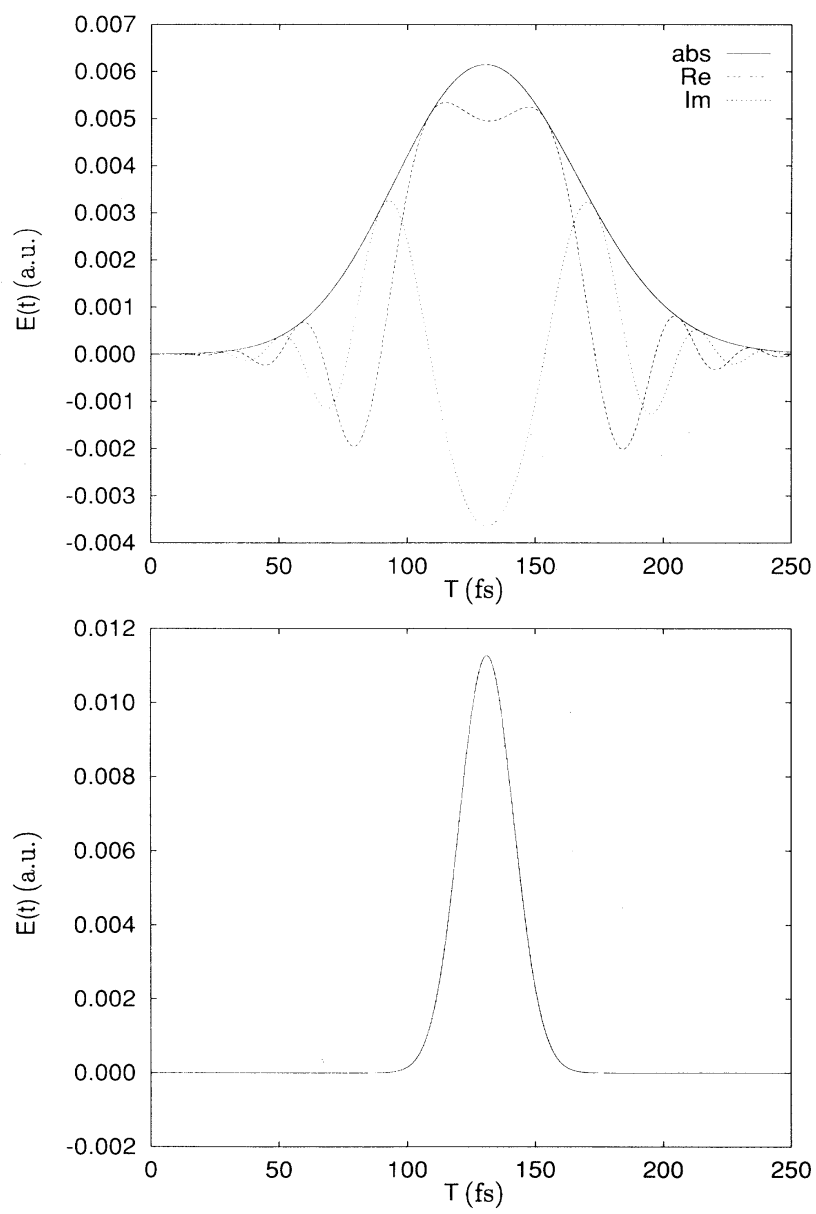

FIG. 3. Optimal chirped electric field (upper graph) for CsI with central frequency removed. Lower plot is for the same electric field except for the chirping parameters are set to 0 . Expressions abs, Re, and Im denote the absolute value and the real and imaginary parts of the complex field, respectively, with removed central frequency.

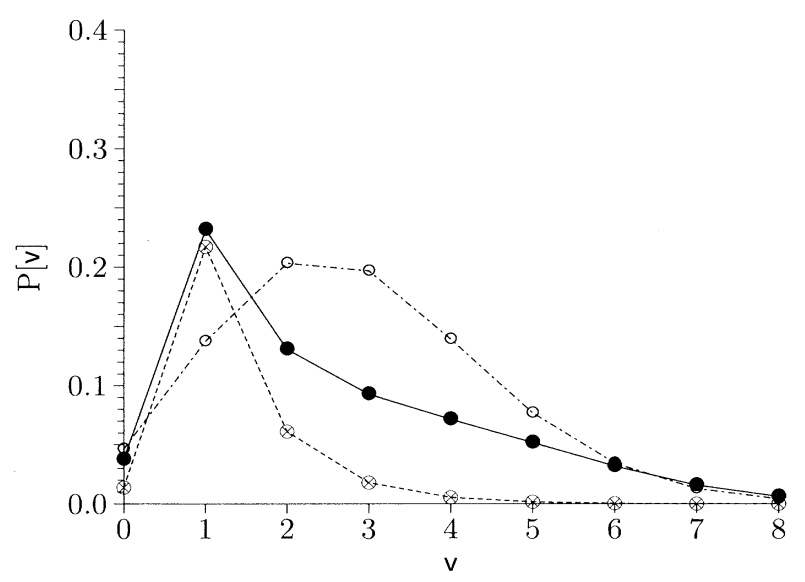

FIG. 4. Final time projections of the ground-state nuclear wave function onto vibrational eigenstates. Solid circles, chirped excitation pulse; crossed circles, nonchirped excitation pulse; open circles, high electric field with no chirp.

BO surfaces are given in Table II. The first optimization was conducted without chirp and it was the amplitude of the electric field that we optimized with a sine squared pulse of $125 \mathrm{fs}$ FWHM.

In the second run the optimization procedure could develop nonzero chirp parameters also. Results of the position probability distribution of the ground- and excitedstate wave functions are given in Fig. 2. The chirped electric field is presented in Fig. 3. The electric field is given with the central frequency removed. The field of the optimization with no chirp is almost an order of magnitude larger, that is, its energy is almost two orders of magnitude larger than that of the optimization with chirp.

The projections of the different final ground electric state wave functions to the vibronic eigenstates of CsI are given in Fig. 4. The solid curve and filled circles is the result of the optimization with chirp. The dashdotted curve and open circles is the result of the almost two orders of magnitude stronger light excitation without chirp. At the expense of high electric-field strength one may achieve similar results as with chirped pulses of moderate strength. The dashed line and crossed circles shows the vibronic content of the excitation when the same moderate electric field was applied but the chirp parameters were set to zero. As it may be seen chirp increases the vibronic content significantly. This fact has been established by Ruhman and Kosloff [45]. The difference between their results and ours is that (1) the chirp we introduced could be produced in the laboratory (they used a different chirp expansion), and (2) these results are the results of an optimization procedure and are the "best" results for a given penalty value $\lambda$. More importantly, the simulated annealing procedure we have followed in the theoretical computation could be followed in the laboratory to optimize the outcome of a given control experiment. 


\section{CONCLUSION}

We have demonstrated that simulated annealing may be an attractive method for solving optimal control of quantum systems. The resulting fields are feasible in the laboratory and the simulated annealing procedure could be followed in the experimental optimization also.

The results presented cannot be considered as the results one should really reach in the laboratory. There are various reasons for that, e.g., we have used models of realistic systems, and we have not taken into account the dependence of the transition dipole moment on the rotational quantum number. However, the approach was designed to open the way to optimal control experiments. Chirp expansion seems very promising from this point of view.

\section{ACKNOWLEDGMENTS}

Part of this work was supported by Brdr. Hartmann's Fond, the Danish Natural Science Research Council, and by OTKA Grant of the Hungarian Academy of Science No. 1890 .
* Electronic address: kebjarne@vm.uni-c.dk

$\dagger$ Electronic address: lorincz@obelix.iki.kfki.hu

[1] D. Tannor and S. Rice, J. Chem. Phys. 83, 5013 (1985).

[2] D. Tannor, R. Kosloff, and S. Rice, J. Chem. Phys. 85, 5805 (1986).

[3] A. Peirce, M. Dahleh, and H. Rabitz, Phys. Rev. A 37, 4950 (1988).

[4] S. Shi, A. Woody, and H. Rabitz, J. Chem. Phys. 88, 6870 (1988).

[5] R. Kosloff et al., Chem. Phys. 139, 201 (1989).

[6] S. Shi and H. Rabitz, Chem. Phys. 139, 185 (1989).

[7] M. Dahleh, A. Peirce, and H. Rabitz, Phys. Rev. A 42, 1065 (1990).

[8] S. Shi and H. Rabitz, J. Chem. Phys. 92, 364 (1990).

[9] S. Shi and H. Rabitz, J. Chem. Phys. 92, 2927 (1990).

[10] S. Tersigni, P. Gaspard, and S. Rice, J. Chem. Phys. 93, 1670 (1990).

[11] K. Yao, S. Shi, and H. Rabitz, Chem. Phys. 150, 373 (1990).

[12] B. Amstrup, R. Carlson, A. Matro, and S. Rice, J. Phys Chem. 95, 8019 (1991).

[13] C. Schwieters and H. Rabitz, Phys. Rev. A 44, 5224 (1991).

[14] J. Beumee and H. Rabitz, J. Chem. Phys. 97, 1353 (1992).

[15] P. Gross, D. Neuhauser, and H. Rabitz, J. Chem. Phys. 96, 2834 (1992).

[16] R. Judson and H. Rabitz, Phys. Rev. Lett. 68, 1500 (1992).

[17] R. Kosloff, A. Hammerich, and D. Tannor, Phys. Rev. Lett. 69, 2172 (1992).

[18] S. Shi and H. Rabitz, J. Chem. Phys. 97, 276 (1992).

[19] B. Amstrup, A. Lörincz, and S. Rice, J. Phys. Chem. 97, 6175 (1993).

[20] P. Gross, D. Neuhauser, and H. Rabitz, J. Chem. Phys. 98, 4557 (1993).

[21] P. Gross et al., Phys. Rev. A 47, 4593 (1993).

[22] V. Kazakov, J. Somlói, and D. Tannor (private communication).

[23] J. Somlói, V. Kazakov, and D. Tannor, Chem. Phys. 172, 85 (1993).

[24] T. Szakács, J. Somlói, and A. Lörincz, Chem. Phys. 172, 1 (1993).

[25] M. Shapiro and P. Brumer, J. Chem. Phys. 84, 4103 (1986).

[26] P. Brumer and M. Shapiro, Faraday Discuss. Chem. Soc. 82, 177 (1986).

[27] P. Brumer and M. Shapiro, Chem. Phys. Lett. 126, 541
(1986).

[28] C. Chan, P. Brumer, and M. Shapiro, J. Chem. Phys. 94, 2688 (1991).

[29] W. Jakubetz, B. Just, J. Manz, and H.-J. Schreier, J. Phys. Chem. 94, 2294 (1990).

[30] W. Jakubetz, J. Manz, and H.-J. Schreier, Chem. Phys. Lett. 165, 100 (1990).

[31] B. Just, J. Manz, and I. Trisca, Chem. Phys. Lett. 193, 423 (1992)

[32] B. Just, J. Manz, and G. Paramonov, Chem. Phys. Lett. 193, 429 (1992).

[33] J. Combariza, S. Görtler, B. Just, and J. Manz, Chem. Phys. Lett. 195, 393 (1992).

[34] G. Paramonov, Chem. Phys. Lett. 169, 573 (1990).

[35] B. Amstrup and N. Henriksen, J. Chem. Phys. 97, 8285 (1992).

[36] N. Scherer et al., J. Chem. Phys. 95, 1487 (1991).

[37] A. Weiner, D. Leaird, J. Patel, and J. R. Wullert II, IEEE J. Quantum Electron. 28, 908 (1992).

[38] A. Weiner, D. Leaird, D. Reitze, and E. Paek, IEEE J. Quantum Electron. 28, 2251 (1992).

[39] D. Reitze, A. Weiner, and D. Leaird, Appl. Phys. Lett. 61, 1260 (1992).

[40] Z. Gogolák, Z. Bor, and G. Szabó, Exp. Tech. Phys. 39, 323 (1991)

[41] M. Nuss, D. Auston, and F. Capasso, Phys. Rev. Lett. 58, 2355 (1987).

[42] A. Siegman, Lasers (University Science Books, Mill Valley, CA, 1986).

[43] S. Kirkpatrick, J. C. D. Gelatt, and M. Vecchi, Science 220, 671 (1983).

[44] W. Press, S. Teukolsky, W. Vetterling, and B. Flannery, Numerical Recipes in $C$, 2nd ed. (Cambridge University Press, New York, 1992).

[45] S. Ruhman and R. Kosloff, J. Opt. Soc. Am. B 7, 1748 (1990).

[46] W. Warren et al., J. Opt. Soc. Am. B 3, 488 (1986).

[47] R. Fork, C. B. Cruz, P. Becker, and C. Shank, Opt. Lett. 12, 483 (1987).

[48] R. Fork, B. Greene, and C. Shank, Appl. Phys. Lett. 38, 671 (1981).

[49] R. Kosloff and H. Tal-Ezer, Chem. Phys. Lett. 127, 223 (1986).

[50] P. van Laarhoven, E. Aarts, and J. Lenstra, Oper. Res. 40, 113 (1992).

[51] C. Leforestier et al., J. Comput. Phys. 94, 59 (1991).

[52] M. Feit, J. J. A. Fleck, and A. Steiger, J. Comput. Phys. 47, 412 (1982). 